\title{
High-speed high-resolution laser diode- based photoacoustic microscopy for in vivo microvasculature imaging
}

\author{
Xiufeng Li®D, Victor T C Tsang, Lei Kang, Yan Zhang and Terence TW Wong*
}

\begin{abstract}
Laser diodes (LDs) have been considered as cost-effective and compact excitation sources to overcome the requirement of costly and bulky pulsed laser sources that are commonly used in photoacoustic microscopy (PAM). However, the spatial resolution and/or imaging speed of previously reported LD-based PAM systems have not been optimized simultaneously. In this paper, we developed a high-speed and high-resolution LD-based PAM system using a continuous wave $L D$, operating at a pulsed mode, with a repetition rate of $30 \mathrm{kHz}$, as an excitation source. A hybrid scanning mechanism that synchronizes a one-dimensional galvanometer mirror and a two-dimensional motorized stage is applied to achieve a fast imaging capability without signal averaging due to the high signal-tonoise ratio. By optimizing the optical system, a high lateral resolution of $4.8 \mu \mathrm{m}$ has been achieved. In vivo microvasculature imaging of a mouse ear has been demonstrated to show the high performance of our LD-based PAM system.
\end{abstract}

Keywords: Photoacoustic microscopy, Laser diode, In vivo imaging, Microvasculature imaging

\section{Introduction}

Photoacoustic tomography is a hybrid imaging modality in which the detected ultrasonic signals are induced by the absorption of pulsed light [1]. Taking the advantage of intrinsic optical absorption contrast, photoacoustic microscopy (PAM) has been widely used to provide high-resolution and label-free images for functional, metabolic, and histological imaging [2-8]. However, the costly and bulky lasers, such as Q-switched diodepumped solid-state laser, Ti:sapphire laser, or optical parametric oscillator laser, are usually required to generate high-energy light pulses with a short pulse width for PA signal generation, preventing the wide usage of PAM system for clinical applications. To address this issue, economical and compact laser diodes (LDs) have been investigated as alternative excitation sources in PAM [9-

\footnotetext{
* Correspondence: ttwwong@ust.hk

Translational and Advanced Bioimaging Laboratory, Department of Chemical and Biological Engineering, Hong Kong University of Science and Technology, Hong Kong, China
}

\section{Springer Open}

14]. However, most of them have limitations of low spatial resolution and/or low imaging speed. For example, a pulsed LD with a wavelength of $905 \mathrm{~nm}$ with a pulse energy of $3 \mu \mathrm{J}$ was used as the excitation source in PAM, achieving a lateral resolution of $7 \mu \mathrm{m}$ [10]. Nevertheless, the pulse repetition rate is relatively low $(1 \mathrm{kHz})$ and 128 times signal averaging is required to improve the signal-to-noise ratio (SNR), limiting the overall imaging speed. Recently, a laser scanning LD-based PAM system was developed without the need for signal averaging [12]. However, the image quality is not satisfactory because of the relatively low lateral resolution $(21 \mu \mathrm{m})$.

Instead of using pulsed LDs, continuous-wave (CW) LDs, which can be overdriven by a pulse driver to generate high-energy light pulses, have also been proposed to be light sources in PAM. Compared to pulsed LDs, although the pulse energy of the pulse-overdriven CW LDs is relatively low $(<250 \mathrm{~nJ})$, they are commercially available in a wide range of wavelengths, ranging from the visible to near-infrared wavelength regions, providing

(c) The Author(s). 2021 Open Access This article is licensed under a Creative Commons Attribution 4.0 International License, which permits use, sharing, adaptation, distribution and reproduction in any medium or format, as long as you give appropriate credit to the original author(s) and the source, provide a link to the Creative Commons licence, and indicate if changes were made. The images or other third party material in this article are included in the article's Creative Commons licence, unless indicated otherwise in a credit line to the material. If material is not included in the article's Creative Commons licence and your intended use is not permitted by statutory regulation or exceeds the permitted use, you will need to obtain permission directly from the copyright holder. To view a copy of this licence, visit http://creativecommons.org/licenses/by/4.0/. 
wavelengths that match the absorption peaks of various absorbers, thus, ensuring sufficient SNR in opticalresolution PAM. Among them, a $450 \mathrm{~nm} \mathrm{CW} \mathrm{LD,} \mathrm{which}$ is pulse-driven by a custom-designed driver to generate light pulses with a pulse energy of $\sim 200 \mathrm{~nJ}$, pulsed width of $\sim 10 \mathrm{~ns}$, and repetition rate of $625 \mathrm{kHz}$, was applied to develop an LD-based PA mesoscope for vasculature imaging [13]. Although the repetition rate is high, the system requires 500 times signal averaging for mouse ear in vivo imaging. Another PA imaging system implementing with a compact fingertip $450 \mathrm{~nm}$ LD also suffers from low resolution and low imaging speed (256 times averaging) [14].

Therefore, there is still a challenge in implementing an LD-based PAM system that can generate high-resolution images with high imaging speed simultaneously. To this end, in this paper, we considered the size of the LD emitter while optimizing the optical system to focus the light onto samples, achieving a high lateral resolution of
$4.8 \mu \mathrm{m}$. With a proper overdrive circuit implementation, high imaging speed is achieved with one-dimensional (1D) galvanometer mirror (GM) scanning that fully utilizes the high repetition rate $(30 \mathrm{kHz})$ of the $\mathrm{LD}$ without the need for signal averaging.

\section{Method}

In this paper, a CW multimode blue LD (L450G1, Thorlabs, Inc.) is used as the excitation source. The light beam emitting from the LD is focused onto the sample by a set of lenses to excite PA signals. Different from solid-state lasers, which are usually considered as ideal light sources, the emitter size of LD has to be considered when designing a high-resolution LD-based PAM system. For the blue LD we employed, the emitter size has a dimension of $1 \times 35 \mu^{2}$ (vertical $\times$ horizontal). Therefore, to achieve high lateral resolution, the horizontal dimension is demagnified to prevent an elliptical focal spot. The optical system that we used to demagnify the
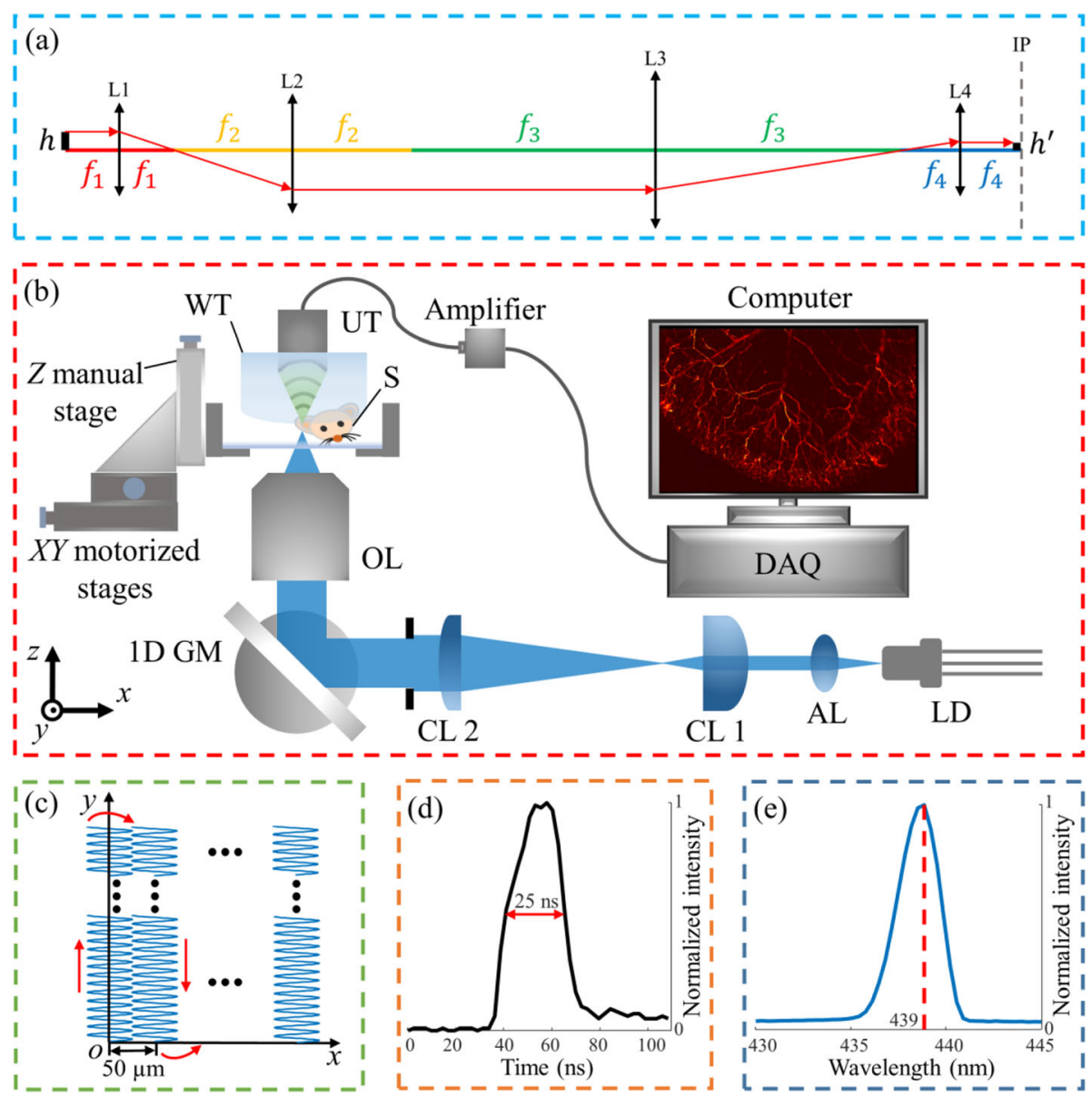

Fig. 1 LD-based PAM system design. a The diagram of the optical system that demagnifies the emitter size of the LD on the image plane; $\mathbf{b}$ The schematic of LD-based PAM; c The scanning trajectory of the system; d The waveform of the LD light pulse, showing a pulse width of $\sim 25 \mathrm{~ns}$; $\mathbf{e}$ The spectrum of the LD light pulse, showing a center wavelength of $\sim 439 \mathrm{~nm}$. L: lens; f: focal length; IP: image plane; $A L$ : aspheric lens; $C L$ : cylindrical lens; OL: objective lens; S: sample; UT: ultrasonic transducer; WT: water tank; DAQ: data acquisition card 
size of the emitter on the image plane is shown in Fig. 1a. According to geometrical optics, the size of the image can be expressed as: $h^{\prime}=\left(f_{4} / f_{3}\right) \cdot\left(f_{2} / f_{1}\right) \cdot h$, where $f_{i}$ is the focal length of the lens $\mathrm{L}_{i}(i=1,2,3,4)$, and $h$ is the size of the object. Thus, by determining the ratio of $\left(f_{4} / f_{3}\right)$ and $\left(f_{2} / f_{1}\right)$, the size of the object on the image plane can be demagnified accordingly, leading to high-resolution imaging with the LD-based PAM system.

Based on the above analysis, we set up a high-speed and high-resolution LD-based PAM system as shown in Fig. 1b. The laser beam emitting from the LD is first collimated by an aspheric lens (A240TM-A, $f_{1}=8 \mathrm{~mm}$, Thorlabs, Inc.). Since the LD has different beam divergence angle $\left(30^{\circ}\right.$ and $6^{\circ}$ in the vertical and horizontal directions, respectively), the laser beam is then expanded along the horizontal direction by a pair of cylindrical lenses (GCL-110114, $f_{2}=25 \mathrm{~mm}$, Daheng Optics; LJ1267RM-A, $f_{3}=250 \mathrm{~mm}$, Thorlabs, Inc.). An iris $(\sim 8$ $\mathrm{mm}$ in diameter) is placed after the second cylindrical lens to control the light beam to a circular shape. The laser beam is then reflected by a $1 \mathrm{D}$ GM before it is focused by an objective lens (LMU-20X-UVB, $f_{4}=9.9 \mathrm{~mm}$, Thorlabs, Inc.) onto the sample for PA signals excitation, achieving rapid scanning with a line-scanning interval of $50 \mu \mathrm{m}$ on the sample. A hybrid scanning that synchronizes 1D optical and two-dimensional mechanical scanning is applied to achieve fast imaging for the whole sample $[7,15]$. The laser-induced PA signals will be detected by a focused ultrasonic transducer (V324-SU, 25 $\mathrm{MHz}$ central frequency, Olympus NDT, Inc.), and amplified by two amplifiers (56 dB, two ZFL-500LN-BNC+, Minicircuit, Inc.). The signals are then filtered by a lowpass filter (BLP-70+, DC-60 MHz, Minicircuit, Inc.) before being collected by a data acquisition card (ATS9350, Alazar Technologies, Inc.). Finally, the signals are processed to reconstruct LD-based PAM images on a computer display.

The details about achieving high imaging speed using 1D GM can be found in our previous work [7]. In brief, as shown in the scanning trajectory (Fig. 1c), the 1D GM repeatedly reflects the incident laser pulses to scan samples along the $x$-axis with a line-scanning interval of $50 \mu \mathrm{m}$, which is smaller than the acoustic focal spot of the ultrasonic transducer to maintain high detection sensitivity. The Y motorized stage [L-509.10SD00, PI (Physik Instrumente) Singapore LLP] is synchronized with the 1D GM and moves along the $y$-axis when the 1D GM finishes a line scan. After the Y motorized stage travels the preset distance, the $\mathrm{X}$ motorized stage [L509.10SD00, PI (Physik Instrumente) Singapore LLP] moves along the $x$-axis with a step size of $50 \mu \mathrm{m}$. The Y motorized stage and the 1D GM system are then synchronized and scan again. The scanning process will stop when the entire sample is scanned completely.
Based on the above parameters of the optical lenses, the theoretical resolution can be estimated as $h^{\prime}=\left(f_{4} / f_{3}\right)$ $\cdot\left(f_{2} / f_{1}\right) \cdot h=(9.9 / 250) \cdot(25 / 8) \cdot 35 \mu \mathrm{m} \approx 4.3 \mu \mathrm{m}$. To generate light pulses, the blue CW LD is driven by a commercially-available pulse driver (PCO-7121, Directed Energy, Inc.) at a repetition rate of $30 \mathrm{kHz}$. The waveform of an emitted light pulse measured with a photodiode detector (PDA10A2, Thorlabs, Inc.) is shown in Fig. 1d, showing a pulse duration of $\sim 25 \mathrm{~ns}$. Figure 1e shows the spectrum of the emitted light measured with an optical spectrometer (USB 2000+, Ocean Optics, Inc.). The center wavelength of the laser is $\sim 439 \mathrm{~nm}$, which is shorter than that in the specification datasheet (center wavelength $\sim 446 \mathrm{~nm}$ ). The spectrum in the datasheet is measured in a CW operating condition with a power of $\sim 3 \mathrm{~W}$, while the power of the pulse-driven LD in this paper is $<5 \mathrm{~mW}$. The observed spectral shift can be accounted for the differences in LD chip temperature as power differs between the $\mathrm{CW}$ operating mode and the pulse-driven mode.

\section{Results \\ Resolution measurement}

To evaluate the spatial resolution of our LD-based PAM system, carbon particles with $1 \mu \mathrm{m}$ in diameter were imaged. An LD-based PAM image of a particle is shown in Fig. 2a. To measure the lateral resolution, the experimental data along the white dotted line (Fig. 2a) was first plotted as the red circles in Fig. $2 b$ and then fitted by a Gaussian function (blue solid line in Fig. 2b). The full width at half maximum (FWHM) of the fitted Gaussian function shows that the lateral resolution of our system is $\sim 4.8 \mu \mathrm{m}$, which is close to the theoretical value $(4.3 \mu \mathrm{m})$.

To measure the axial resolution, we applied the Hilbert transform to extract the envelope of the A-line PA signal of the carbon particle at the central position, shown in Fig. 2c. The FWHM of the envelope is $\sim 47 \mathrm{~ns}$, which represents $\sim 70 \mu \mathrm{m}$ in axial resolution by assuming the speed of sound to be $\sim 1480 \mathrm{~m} / \mathrm{s}$.

\section{Leaf phantom imaging}

To demonstrate the imaging capability of our system, a leaf phantom dyed with black ink was imaged. Figure 3a shows a photograph of the leaf phantom, and Fig. 3b shows the LD-based PAM image of the red marked region in Fig. 3a. We can see from Fig. 3 that the structure of the leaf in the LD-based PAM (Fig. 3b) image shows high similarity with that in the photograph (Fig. 3a). The maximum SNR can reach up to $\sim 42 \mathrm{~dB}$ with a pulse energy of $\sim 30 \mathrm{~nJ}$. The field-of-view (FOV) of the LD-based PAM image (Fig. $3 \mathrm{~b}$ ) is $10 \times 10 \mathrm{~mm}^{2}$. With this superior SNR, no signal averaging is required. With the high repetition rate $(30 \mathrm{kHz})$ of the $\mathrm{LD}$ and fast scanning 

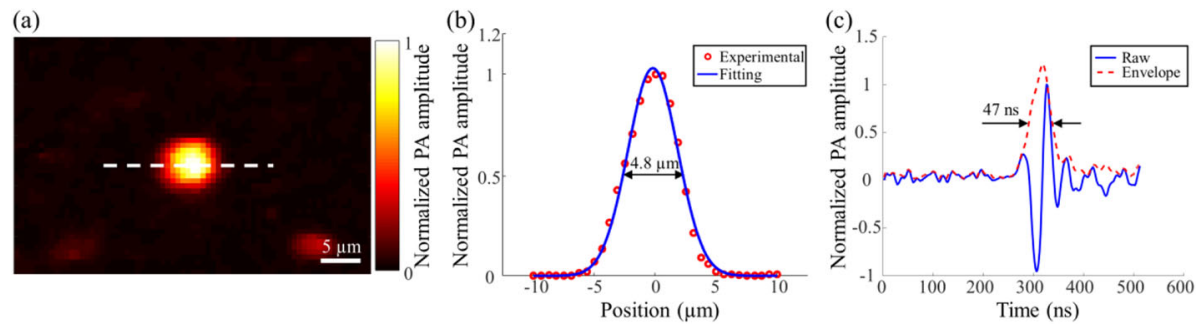

Fig. 2 Spatial resolution measurement. a An LD-based PAM image of a carbon particle; $\mathbf{b}$ Lateral resolution measurement. The red circles are the experimental data along the white dotted line in Fig. $\mathbf{a}$. The blue solid line is a Gaussian fitting function; c Axial resolution measurement. The red dotted line represents the envelope of the A-line signal of the central position of the carbon particle in Fig. a

mechanism, the total imaging time for this large FOV is $\sim 28.5$ minutes with a scanning step size of $1.3 \mu \mathrm{m}$ in the $x$-axis, and $1.6 \mu \mathrm{m}$ in the $y$-axis (corresponding to 32 points on each line-scanning interval using GM scanning). According to our current lateral resolution ( $4.8 \mu \mathrm{m}$ ), a larger scanning step can be set to further improve the imaging speed without sacrificing the imaging resolution.

\section{In vivo microvasculature imaging of a mouse ear}

To verify the potential of our LD-based PAM system for in vivo microvasculature imaging, a mouse ear was imaged. The protocol of animal experiments was approved by the Animal and Plant Care Facility at The Hong Kong University of Science and Technology. The mouse was maintained anesthetized with $2 \%$ isoflurane mixed with oxygen at a flow rate of $0.8 \mathrm{~L} / \mathrm{min}$ during the experiment. An LD-based PAM image of the mouse ear acquired by our system is shown in Fig. 4a, where the distribution of microvasculature networks can be clearly observed. The SNR can reach up to $31 \mathrm{~dB}$ with a pulse energy of $\sim 60 \mathrm{~nJ}$. The optical fluence on the tissue surface is $\sim 0.8 \mathrm{~mJ} / \mathrm{cm}^{2}$, which is lower than the American National Standards Institute safety limit of $20 \mathrm{~mJ} / \mathrm{cm}^{2}$
[16]. The FOV is $8 \times 5 \mathrm{~mm}^{2}$. The image acquisition time is $\sim 11.5$ minutes. Figures $4 \mathrm{~b}$ and $\mathrm{c}$ show the close-up images of the blue and green marked regions in Fig. $4 \mathrm{a}$, respectively. The microvasculature can be clearly resolved. These promising results evaluate that our LDbased PAM system can provide high-quality images for in vivo microvasculature imaging at high speed.

\section{Discussions and conclusions}

In this paper, we developed a high-resolution and efficient LD-based PAM system by optimizing the parameters of the optical system design according to the emitter size of the LD. Moreover, the high SNR allows no signal averaging, and thus a fast optical scanning mechanism can be used to improve the overall imaging speed. To the best of our knowledge, it is the first demonstration of an LD-based PAM system that can achieve high resolution $(<5 \mu \mathrm{m})$ and high imaging speed $(30 \mathrm{kHz}$ A-line rate) simultaneously. Nevertheless, the performance of our system can be further improved. First, the focal lengths of the two-cylindrical lenses are relatively large, which is less suitable for a more compact system. A pair of cylindrical lenses with shorter focal lengths, while maintaining the equivalent focal length ratio can

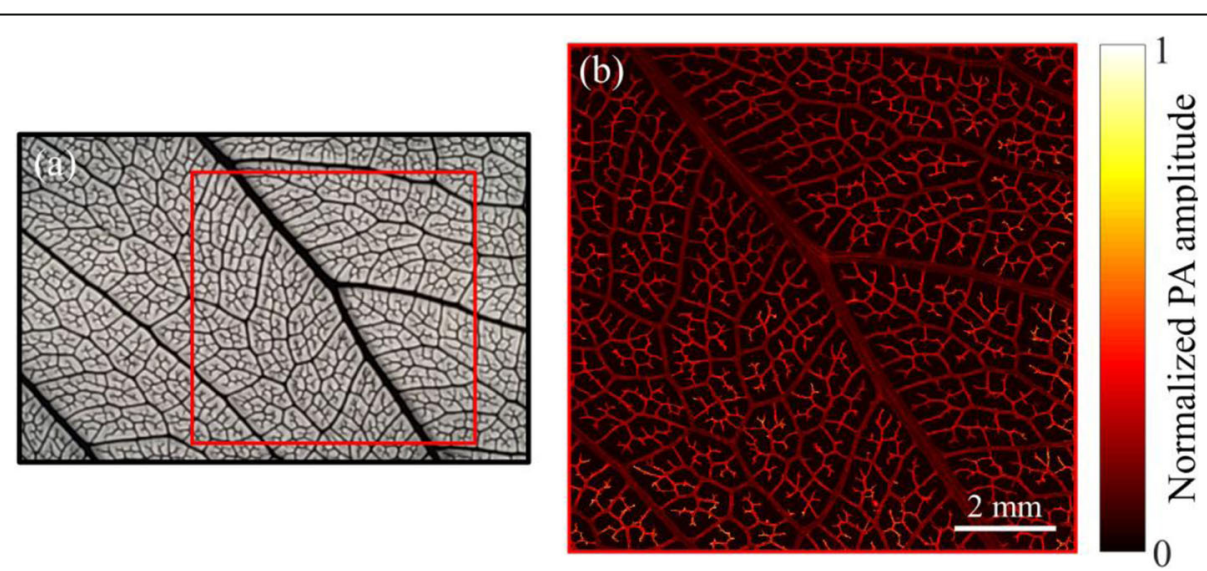

Fig. 3 Leaf phantom imaging. a A photograph of a leaf phantom dyed with black ink; b An LD-based PAM image of the red marked region in Fig. a 

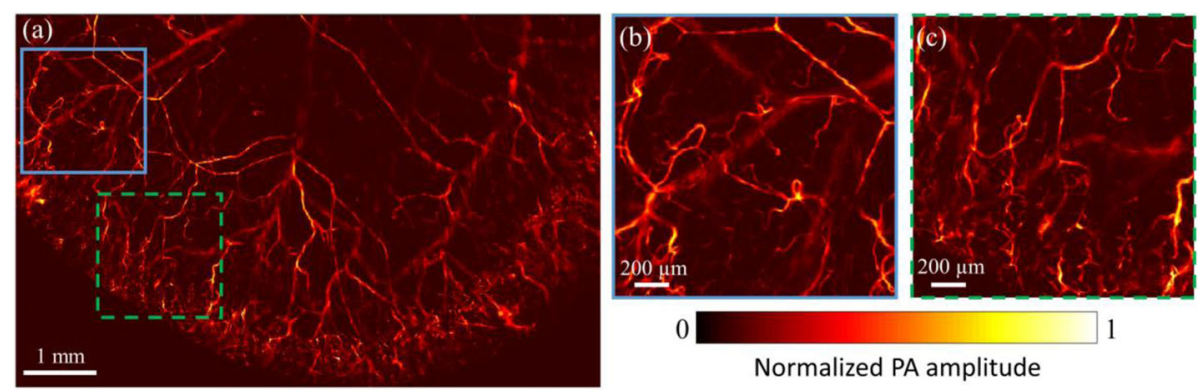

Fig. 4 a In vivo LD-based PAM imaging of a mouse ear. $\mathbf{b}$ and $\mathbf{c}$ Close-up images of the blue and green marked regions in Fig. a

be used. Second, the current system is in transmission mode, which limits its applications to only imaging thin tissue samples, e.g., mouse ear in vivo (Fig. 4). To promote the LD-based PAM system with more applications, a ring-shaped ultrasonic transducer [17] or other optically transparent ultrasonic transducers [18] can be used to develop a reflection-mode LD-based PAM system. Third, the size of the pulse driver can be further minimized to make the system more compact. A compact, low-cost, and high-performance LD-based PAM system will be beneficial for various biomedical applications, and is expected to be more accessible for clinical imaging.

The stability of the pulse energy of pulse-driven CW LDs has been investigated in ref. [13]. We also tested our LD-based PAM system for more than 24hours under a repetition rate of $30 \mathrm{kHz}$ with a pulse energy of $100 \mathrm{~nJ}$ (on the sample plane). No damage or degradation of optical energy has been observed, proving the high stability of the pulse-driven CW LD.

In conclusion, we developed a high-speed and highresolution LD-based PAM system for microvasculature imaging. The light beam is tightly focused on the sample by an optimized demagnifying optical system, achieving a high lateral resolution of $4.8 \mu \mathrm{m}$. Our system does not require any signal averaging, thus, enabling high imaging speed with the integration of a 1D GM. The in vivo mouse ear imaging demonstrated that our system could provide high-quality images for microvasculature at high imaging speed.

\section{Abbreviations}

PAM: Photoacoustic microscopy; LD: Laser diode; SNR: Signal-to-noise ratio; CW: Continuous wave; 1D: One-dimensional; GM: Galvanometer mirror;

FWHM: Full width at half maximum; FOV: Field-of-view

\section{Acknowledgments}

The authors thank Jiangbo Chen of City University of Hong Kong for helping measure the spectrum of the laser diode.

\section{Funding}

Hong Kong Innovation and Technology Commission, No. ITS/036/19; Research Grants Council of the Hong Kong Special Administrative Region, No. 26203619.

\section{Availability of data and materials}

The datasets used and analyzed during the current study are available from the corresponding author on reasonable request.

\section{Competing interests}

The authors declare no conflicts of interest.

Received: 30 October 2020 Accepted: 22 December 2020

Published online: 11 January 2021

\section{References}

1. Wang LV, Hu S (2012) Photoacoustic tomography: in vivo imaging from organelles to organs. Science 335:1458-1462. https://doi.org/10.1126/ science.1216210

2. Yao J, Wang L, Yang J-M, Maslov KI, Wong TTW, Li L, Huang C-H, Zou J, Wang LV (2015) High-speed label-free functional photoacoustic microscopy of mouse brain in action. Nat Methods 12:407-410. https://doi.org/10.1038/ nmeth.3336

3. Li M, Chen J, Wang L (2020) High acoustic numerical aperture photoacoustic microscopy with improved sensitivity. Opt Lett 45:628. https://doi.org/10.1364/ol.384691

4. Lan B, Liu W, Wang Y, Shi J, Li Y, Xu S, Sheng H, Zhou Q, Zou J, Hoffmann U, Yang W, Yao J (2018) High-speed widefield photoacoustic microscopy of small-animal hemodynamics. Biomed Opt Express 9:4689. https://doi.org/10. 1364/BOE.9.004689

5. Liu C, Liang Y, Wang L (2019) Optical-resolution photoacoustic microscopy of oxygen saturation with nonlinear compensation. Biomed Opt Express 10: 3061. https://doi.org/10.1364/BOE.10.003061

6. Yao J, Maslov KI, Zhang Y, Xia Y, Wang LV (2011) Label-free oxygenmetabolic photoacoustic microscopy in vivo. J Biomed Opt 16:076003. https://doi.org/10.1117/1.3594786

7. Li X, Kang L, Zhang Y, Wong TTW (2020) High-speed label-free ultraviolet photoacoustic microscopy for histology-like imaging of unprocessed biological tissues. Opt Lett 45:5401. https://doi.org/10.1364/OL.401643

8. Chen J, Zhang Y, He L, Liang Y, Wang L (2020) Wide-field polygon-scanning photoacoustic microscopy of oxygen saturation at 1-MHz A-line rate. Photoacoustics 20:100195. https://doi.org/10.1016/j.pacs.2020.100195

9. Zeng L, Liu G, Yang D, Ji X (2013) Portable optical-resolution photoacoustic microscopy with a pulsed laser diode excitation Appl Phys Lett 102: https:// doi.org/10.1063/1.4791566

10. Wang T, Nandy S, Salehi HS, Kumavor PD, Zhu Q (2014) A low-cost photoacoustic microscopy system with a laser diode excitation. Biomed Opt Express 5:3053 https://doi.org/10.1364/boe.5.003053

11. Zeng L, Piao Z, Huang S, Jia W, Chen Z (2015) Label-free optical-resolution photoacoustic microscopy of superficial microvasculature using a compact visible laser diode excitation. Opt Express 23:31026 https:/doi.org/10.1364/ oe. 23.031026 
12. Erfanzadeh M, Kumavor PD, Zhu Q (2018) Laser scanning laser diode photoacoustic microscopy system. Photoacoustics 9:1-9. https://doi.org/10. 1016/j.pacs.2017.10.001

13. Stylogiannis A, Prade L, Buehler A, Aguirre J, Sergiadis G, Ntziachristos V (2018) Continuous wave laser diodes enable fast optoacoustic imaging. Photoacoustics 9:31-38. https://doi.org/10.1016/j.pacs.2017.12.002

14. Zhong H, Zhang J, Duan T, Lan H, Zhou M, Gao F (2019) Enabling both time-domain and frequency-domain photoacoustic imaging by a fingertip laser diode system. Opt Lett 44:1988. https://doi.org/10.1364/OL.44.001988

15. Li L, Yeh C, Hu S, Wang L, Soetikno BT, Chen R, Zhou Q, Shung KK, Maslov Kl, Wang LV (2014) Fully motorized optical-resolution photoacoustic microscopy. Opt Lett 39:2117-2120. https://doi.org/10.1364/OL.39.002117

16. American National Standards Institute Inc (ANSI) (2007) ANSI Z136.1-2007: American National Standard for Safe Use of Lasers.

17. Wong TTW, Zhang R, Hai P, Zhang C, Pleitez MA, Aft RL, Novack D V., Wang L V. (2017) Fast label-free multilayered histology-like imaging of human breast cancer by photoacoustic microscopy. Sci Adv 3. https://doi.org/10. 1126/sciadv. 1602168

18. Tsang VTC, Li X, Wong TTW (2020) A review of endogenous and exogenous contrast agents used in Photoacoustic tomography with different sensing configurations. Sensors 20:5595. https://doi.org/10.3390/s20195595

\section{Publisher's Note}

Springer Nature remains neutral with regard to jurisdictional claims in published maps and institutional affiliations.

\section{Submit your manuscript to a SpringerOpen ${ }^{\circ}$ journal and benefit from:}

- Convenient online submission

- Rigorous peer review

- Open access: articles freely available online

- High visibility within the field

- Retaining the copyright to your article

Submit your next manuscript at $\boldsymbol{\nabla}$ springeropen.com 